\title{
Thermal Conductivity of Cross-linked Polymers. A Comparison between Measured and Calculated Thermal Conductivities
}

\author{
Osamu Yамамото and Hirotaro Kambe \\ Polymer Research Division, Department of Materials, Institute of \\ Space and Aeronautical Science, University of Tokyo, \\ Komaba, Meguro-ku, Tokyo, Japan.
}

(Received February 22, 1971)

\begin{abstract}
The thermal conductivity of the following cross-linked polymers was studied: (1) polystyrene cross-linked with divinylbenzene, (2) poly(methyl methacrylate) cross-linked with triallylcyanurate, (3) irradiated high-pressure polyethylene, and (4) epoxy resin cured with aliphatic amine. The thermal conductivity was found to increase with an increasing degree of cross-linking. The results were compared with calculated values obtained previously on the basis of the theory. The temperature dependence of the thermal conductivity was also discussed using the derived theory.
\end{abstract}

KEY WORDS Thermal Conductivity $;$ Cross-linking / Glass Transition Temperature / Irradiation / Polystyrene / Poly(methyl methacryllate) / Polyethylene / Epoxy Resin / Temperature Dependence /

Previous studies on thermal conductivity have mainly discussed linear amorphous polymers and there are few reports concerning the thermal conductivity of cross-linked polymers. However, the effect of cross-linking is important as well as other factors affecting their thermal conductivity, because the information obtained from the cross-linked polymers indicates an interpretation for the thermal conduction of amorphous polymers.

Tomlinson, et al., ${ }^{1}$ pointed out that the thermal conductivity of irradiated polyethylene above the melting temperature increases with an increasing radiation dose. Irradiation of polyethylene makes cross-links between polymer chains, so that the result indicates the effect of radiation induced cross-linking on the thermal conductivity. Recently, Knappe and Yamamoto ${ }^{2}$ have measured the thermal conductivity of crosslinked polymers (poly(methyl methacrylate) crosslinked with triallylcyanurate and irradiated highpressure polyethylene) and found that the thermal conductivity increases with an increasing degree of cross-linking. More recently, Ozawa and Kanari ${ }^{3}$ have measured the thermal conductivity of epoxy resins cured with different aliphatic amines. They also found that cross-linking increases the thermal conductivity of the epoxy resins.
The present paper is concerned with the thermal conductivity of these cross-linked polymers and polystyrene cross-linked with divinylbenzene. The results were compared with calculated values obtained previously ${ }^{4}$ on the basis of the theory.

\section{EXPERIMENTAL}

\section{Polystyrene Cross-linked with Divinylbenzene $(S T-D V B)$}

The copolymerization of styrene with divinylbenzene was carried out at $70^{\circ} \mathrm{C}$ for $24 \mathrm{hr}$. Benzoylperoxide used as a catalyst was $1.0 \%$ by weight of the total monomer. After polymerization at $70^{\circ} \mathrm{C}$, the sample was further post-cured at $130^{\circ} \mathrm{C}$ for $3 \mathrm{hr}$. The contents of the divinylbenzene in a series of these copolymers was $0,5,10$, and $20 \%$ by weight. The specimen for thermal conductivity measurements was turned on a lathe to a cylinder of outer diameter of $16 \mathrm{~mm}$ and inner diameter of $5 \mathrm{~mm}$.

The apparatus with cylindrical symmetry used in the determination of the thermal conductivity of the ST-DVB copolymer is identical to that previously described. ${ }^{5}$ Measurements were made in the temperature range from 30 to $130^{\circ} \mathrm{C}$.

Volume measurements for the ST-DVB copolymer were carried out with the so-called Bekkedahl type dilatometer with a capillary 


\section{O. Үамамото and H. КАмве}

having a diameter of $1.0 \mathrm{~mm}$. The cylindrical sample (about $0.5-1.0 \mathrm{~g}$ ) was placed in the bulb of a Pyrex dilatometer. After evacuating the bulb, the bulb was filled with mercury. Volume change was measured by reading the level of mercury in the capillary. The heating rate used here was about $0.4^{\circ} \mathrm{C} / \mathrm{min}$.

\section{RESULTS AND DISCUSSION}

\section{Polystyrene Cross-linked with Divinylbenzene $(S T-D V B)$}

The temperature dependence of the thermal conductivity for ST-DVB copolymers is presented in Figure 1. For uncross-linked polystyrene, a break in the temperature curve is found in the glass transition range $\left(85-90^{\circ} \mathrm{C}\right)$. In a series of cross-linked polystyrenes, the break moves to higher temperatures with an increasing content of DVB. Also it was found that the temperature coefficient of the thermal conductivity in the glass transition range becomes smaller with the increasing content of DVB, and hence the break is less remarkable. These results can be discussed by specific volume measurements. Figure 2 shows the volume-temperature curves of the ST-DVB copolymers. Each curve is represented by two intersecting

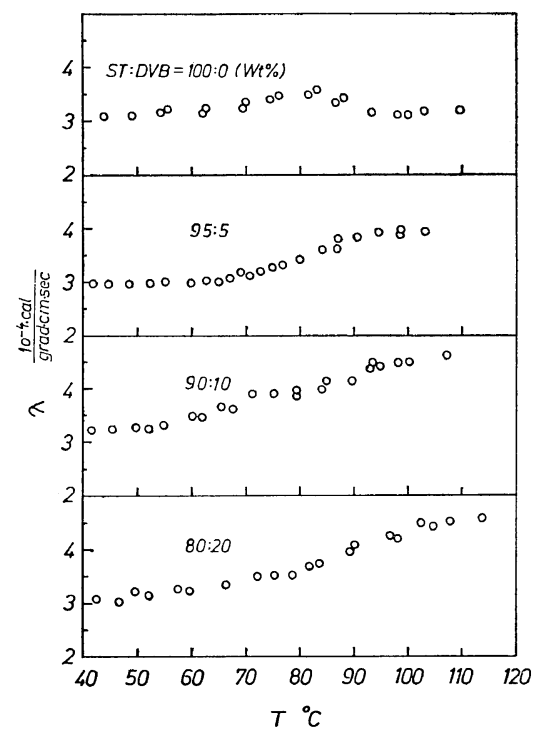

Figure 1. Thermal conductivity of polystyrene cross-linked with divinylbenzene at contents of 0 , 5 , 10 , and $20 \%$ by weight.

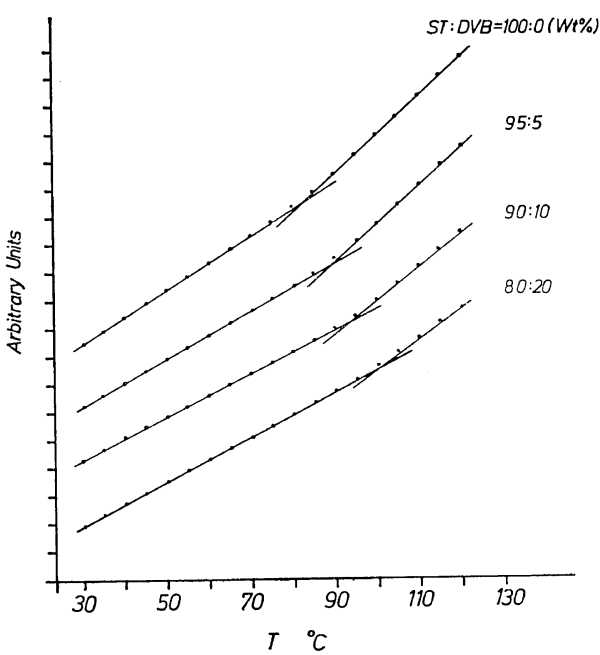

Figure 2. Volume-temperature curves for crosslinked polystyrene.

Table I. $T_{\mathrm{g}}$ and $M_{\mathrm{c}}$ of cross-linked polystyrene

\begin{tabular}{ccccc}
\hline & \multicolumn{4}{c}{ DVB } \\
\cline { 2 - 5 } & $0 \mathrm{wt} \%$ & $5 \mathrm{wt} \%$ & $10 \mathrm{wt} \%$ & $20 \mathrm{wt} \%$ \\
\hline$T_{\mathrm{g}},{ }^{\circ} \mathrm{C}$ & 82 & 88 & 92 & 100 \\
$M_{\mathrm{c}}, \mathrm{g} / \mathrm{mol}$ & - & $6.2 \times 10^{3}$ & $3.7 \times 10^{3}$ & $2.0 \times 10^{3}$ \\
\hline
\end{tabular}

straight lines, where the glass transition temperature $T_{\mathrm{g}}$ is at the intersection of the two lines. $T_{\mathrm{g}}$ values obtained in this way are listed in Table I. The result shows that $T_{\mathrm{g}}$ increases with an increasing content of DVB. At $20 \%$ by weight of DVB, the shift of $T_{g}$ was observed to be about $20^{\circ} \mathrm{C}$. Similar change has been reported by Ueberreiter and Kanig. ${ }^{6}$

The effect of cross-linking on the thermal conductivity may be discussed in the following way. Recently, Knappe, et al., ${ }^{7}$ have measured the thermal conductivity of copolymers or homopolymers with side chains of different lengths. They found that the thermal conductivity of these polymers, when plotted against the temperature difference of $\left(T-T_{\mathrm{g}}\right)$, behaved in the expected manner. A typical example can be seen in the polymers of the methacrylate series, where the thermal conductivity plotted against the temperature difference was found to increase with increasing length of the side chain. The physical meaning of the reduced 


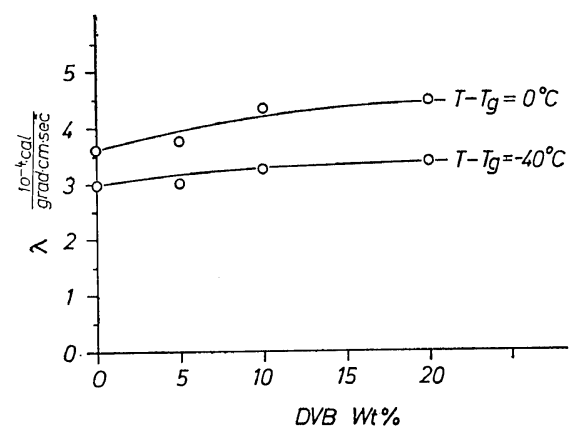

Figure 3. Thermal conductivity of cross-linked polystyrene.

temperature may be given as follows. At the high-temperature region, the main factors characterizing the behavior of the thermal conductivity are the specific heat and the density, as discussed previously. The specific heat relative to the thermal conductivity increases slightly with an increasing temperature without any remarkable change at $T_{\mathrm{g}}$. The slight increase of the specific heat may relate to the anharmonicity of forces between chains ${ }^{8}$ and all vibrations contributing to the specific heat seem to be excited in this high-temperature region. On the other hand, the density of polymer decreases slowly with increasing temperature, but at $T_{\mathrm{g}}$ an abrupt decrease occurs. This arises from the spontaneous increase of the free volume at $T_{\mathrm{g}}$ and prevents the effective propagation of heat, so that a decrease of the thermal conductivity is observed. According to the free volume theory, ${ }^{9}$ the free volume fraction of polymers has a value close to 0.025 up to the glass transition temperature. This leads us to compare the thermal conductivity of various amorphous polymers by shifting it by $\left(T-T_{\mathrm{g}}\right)$ along the temperature axis. Figure 3 shows the change of the reduced thermal conductivity of the ST-DVB copolymer against the DVB content at two temperatures $\left(T-T_{\mathrm{g}}=0,-40^{\circ} \mathrm{C}\right)$. From this Figure, thermal conductivity is found to increase with an increasing content of DVB and the effect of cross-linking becomes slightly larger with increasing temperature. The effect of cross-linking on thermal conductivity can be discussed by using eq 7 or eq 8 of a previous paper, ${ }^{4}$ if the cross-linking parameter $X$ is

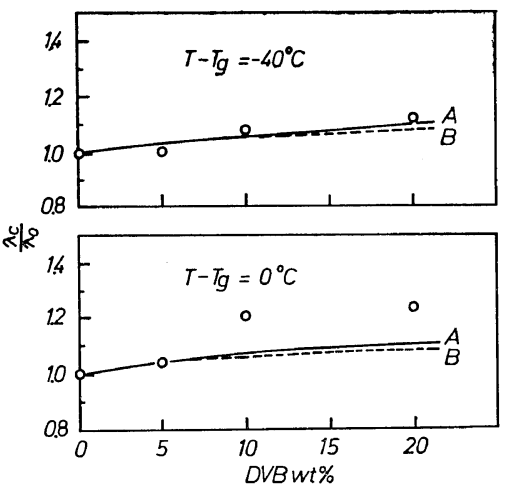

Figure 4. Thermal conductivity of cross-linked polystyrene: $\bigcirc$, experimental; A, calculated from $\lambda_{\mathrm{c}} / \lambda_{0}=1+4.3 X ; \quad \mathrm{B}$, calculated from $\lambda_{\mathrm{c}} / \lambda_{0}=1+$ $\left\{\left(P^{2}-P\right) /(2 P+1)\right\} X$.

known.

Is is well known that cross-linking increases the glass transition temperature. For some crosslinked polymers, it is possible to calculate the molecular weight of segments between cross-links $M_{\mathrm{c}}$ from the observed shift in $T_{\mathrm{g}}$. Recently, Nielsen ${ }^{10}$ has derived an equation relating the shift in $T_{\mathrm{g}}$ to the degree of cross-linking for the ST-DVB copolymer. The original equation was taken from that presented by DiMarzio and DiBenedetto, who had discussed the shift of $T_{\mathrm{g}}$ due solely to cross-linking. The equation given by Nielsen is as follows

$$
T_{\mathrm{g}}-T_{\mathrm{g} 0}=\frac{2 T_{\mathrm{g} 0}}{n_{\mathrm{c}}}
$$

where $T_{\mathrm{g} 0}$ is the glass transition temperature of the uncross-linked polymer, $T_{\mathrm{g}}$ is the cross-linked polymer and $n_{\mathrm{c}}$ is the average number of the carbon atoms in the polymer backbone between cross-links. Then $M_{\mathrm{c}}\left((1 / 2) n_{\mathrm{c}} \times\right.$ molecular weight of styrene) was calculated by using eq 1 with the values determined from volume measurements. The results are also listed in Table I. Figure 4 shows the results for the calculated and measured thermal conductivities. The data are satisfactorily represented by the calculated values.

Poly(methyl methacrylate) Cross-linked with Triallylcyanurate (MMA-TAC)

The thermal conductivity of MMA-TAC copolymers has been measured by Knappe and 


\section{O. Yamamoto and H. Kambe}

Yamamoto. ${ }^{2}$ The result shows that the thermal conductivity of these polymers, similar to that of the ST-DVB copolymers, increases slightly with an increasing content of TAC. The break was also observed at the glass transition temperature and the temperature of this break is shifted to higher temperatures with an increasing content of TAC. At the TAC content of $10 \%$ by weight, the shift of $T_{\mathrm{g}}$ was observed to be $12^{\circ} \mathrm{C}$. The temperature dependence of the thermal conductivity below $T_{\mathrm{g}}$ has the same tendency for all samples. On the other hand, it increases with an increasing content of TAC above $T_{\mathrm{g}}$ and the break becomes less remarkable, reflecting the change of the thermal expansion of cross-linked polymers.

For some cross-linked polymers, we must take notice of the copolymer effect. In this crosslinked system, TAC is considered as a copolymerizing unit. However, if the copolymer effect exists, the resulting effect would be that expected for the cyanurate ring. This, as a side group, seems to cause a decrease of the thermal conductivity. Indeed, the thermal conductivity of heat-treated acrylonitrile-methyl methacrylate copolymer apparently decreases with the formation of the glutaroimide ring. ${ }^{2}$ The increased thermal conductivity with an increasing content of TAC must arise from the cross-linking effect. Figure 5 shows the result expressed as the relation of the reduced thermal conductivity against

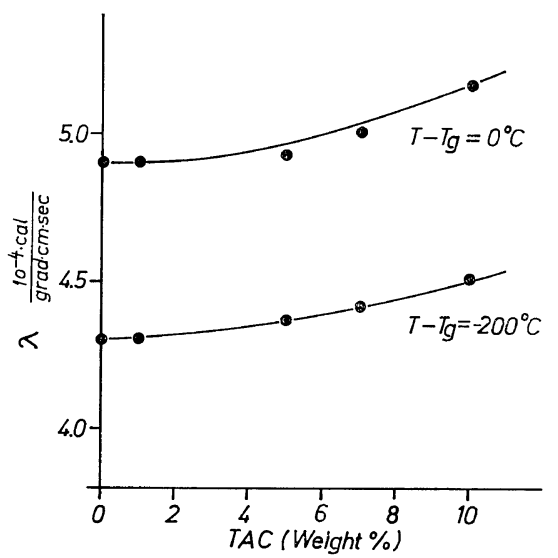

Figure 5. Thermal conductivity of poly(methyl methacrylate) cross-linked with triallylcyanurate at contents of $0,1,5,7$, and $10 \%$ by weight.

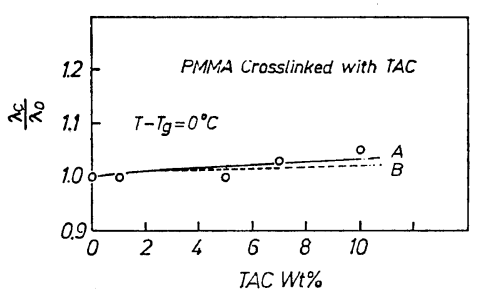

Figure 6. Thermal conductivity of crosslinked poly(methyl methacrylate): $\bigcirc$, experimental; $A$, calculated from $\lambda_{\mathrm{c}} / \lambda_{0}=1+4.3 X ; \mathrm{B}$, calculated from $\lambda_{\mathrm{c}} / \lambda_{0}=1+\left\{\left(P^{2}-P\right) /(2 P+1)\right\} X$.

the content of TAC. To discuss the increased thermal conductivity, $M_{\mathrm{c}}$ values determined from the swelling equilibrium ${ }^{2}$ were used to calculate the parameter $X$. For the MMA-TAC copolymer, $M_{\mathrm{g}}$ was taken as $50 \mathrm{~g} / \mathrm{mol}$ of the molecular weight of a chain unit. Figure 6 shows the measured and calculated thermal conductivities. The solid line indicating the calculated values agrees fairly well with the measured ones.

\section{Irradiated High Pressure Polyethylene}

Figure 7 shows the plot of the thermal conductivity of irradiated high-pressure polyethylene at three different temperatures. The original result was also quoted from the published data. ${ }^{2}$ The thermal conductivity was found to decrease with the radiation dose up to $600 \mathrm{MRD}$ in the temperatures below the melting point. For the sample irradiated to $1000 \mathrm{MRD}$, the thermal conductivity decreases further in the low-temperature region, but near the melting point the values, as a consequence of the cross-linking

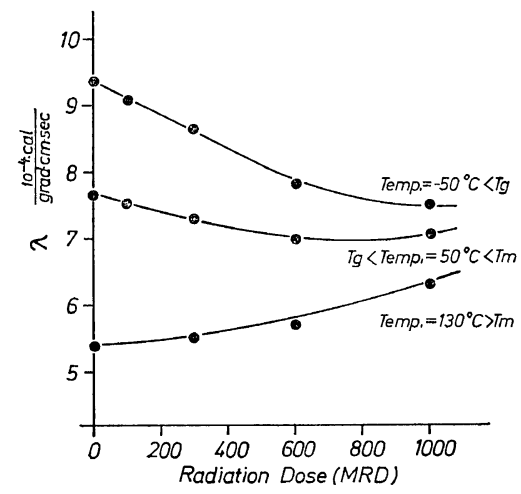

Figure 7. Effect of irradiation on the thermal conductivity of high-pressure polyethylene. 
effect, are higher than those of the less-irradiated samples.

In the temperature region above the melting point, the thermal conductivity for all samples increases with the radiation dose. Tomlinson, et al. ${ }^{1}$ also reported a similar change of thermal conductivity in the molten state. In this temperature region, crystallites are comletely destroyed and the increased thermal conductivity results from radiation-induced cross-linking, where cross-linking takes place between two different chains. The effect of cross-linking by irradiation of polyethylene has been observed in some physical properties in the molten state. Elastic modulus increases with the radiation dose $^{11}$ and heavily irradiated polyethylene exhibits rubber-like elasticity. The density of polyethylene above the melting point also increases with the radiation dose, which binds polymer chains more closely together. ${ }^{11}$ In some polymers irradiation produces chain scission as well as cross-linking. However, for polyethylene, Alexander, Toms, ${ }^{12}$ and Schumacher ${ }^{13}$ reported that $\mathrm{C}-\mathrm{C}$ bond scission is of little importance in the process. To compare the calculated thermal conductivity with the measured one, the degree of cross-linking was estimated using a $G$ value of cross-linking. ${ }^{14}$ The $G$ value is expressed by

$$
G=0.48 \times 10^{6} q_{0} / w
$$

where $q_{0}$ corresponds to the cross-linked portion of a $\mathrm{CH}_{2}$ unit per MRD, if $w$ is taken as the molecular weight of the $\mathrm{CH}_{2}$ unit. Chapiro ${ }^{11}$ collected the $G$ values determined by various investigators and reported that the most probable value of $G$ at room temperature is $G=2.0 \pm 0.4$. However, recently Kang, et al. ${ }^{15}$ Fallgatter, and Dole $^{16}$ have found different values at room tem-

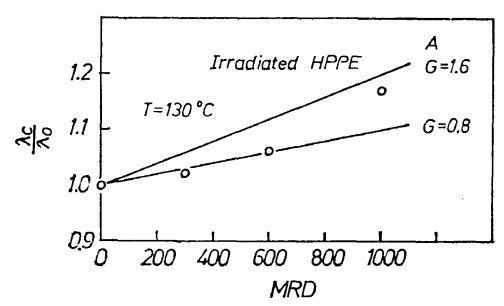

Figure 8. Tnermal conductivity of irradiated high-pressure polyethylene: $\bigcirc$, experimental; $A$, calculated from $\lambda_{c} / \lambda_{0}=1+4.3 X$. perature from various experiments necessary for the determination of the $G$ value. The new values reported by them are in the range of $0.8-1.0$ at low irradiation doses and approach a value of 1.5 at high doses. In our case the values $G=0.8-1.6$ were taken. The degree of cross-linking calculated with eq 2 directly corresponds to the parameter $X$. Thus the comparison between the calculated and measured thermal conductivities can be made with eq 8 of the previous paper. ${ }^{4}$ It can be found from Figure 8 that the thermal conductivity data satisfactorily explain the tendency of the $G$ value to be as determined by Kang, et al., Fallgatter, and Dole.

Epoxy Resin Cured with Aliphatic Amine

Some further evidence in support of the theory is given in Figure 9, which has been measured

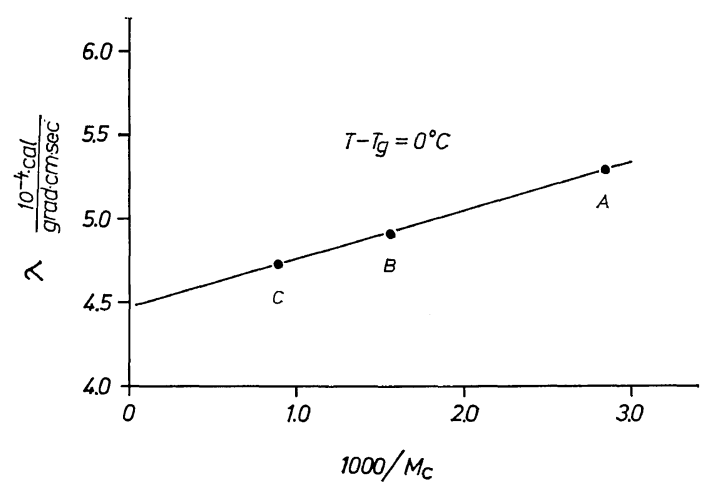

Figure 9. Thermal conductivity of epoxy resin cured with aliphatic amine. Molar ratio of epoxy prepolymer to ethylenediamine and $n$-octylamine: A, $100: 50: 0$; B, $100: 33: 33$; C, $100: 25: 50$.

by Ozawa and Kanari for the thermal conductivity of epoxy resins cured with aliphatic amines (Epon 828 cured with ethylene-diamine and $n$-octylamine). The results show the same behavior as obtained for ST-DVB and MMATAC copolymers. A linear relationship is also found between the reduced thermal conductivity and the degree of cross-linking $1 / M_{\mathrm{c}}$. In this case $M_{\mathrm{c}}$ was estimated from measurements of shear modulus. Although direct comparison with the theory is not possible, the theory explains the data of Figure 9. From eq 8 of a previous paper, ${ }^{4}$ the thermal conductivity of a 
cross-linked polymer is given as follows

$$
\lambda_{\mathrm{c}}=\lambda_{0}+\frac{\text { Constant }}{M_{\mathrm{c}}}
$$

which predicts the linear relationship between $\lambda_{\mathrm{c}}$ and $1 / M_{\mathrm{c}}$.

\section{REFERENCES}

1. J. N. Tomlinson, D. E. Kline, and J. A. Sauer, SPE Transactions, 5, 44 (1965).

2. W. Knappe and O. Yamamoto, Kolloid-Z. Z. Polym., 240, 775 (1970).

3. T. Ozawa and K. Kanari, Preprint, SPSJ 19th Symposium on Macromolecules, Tokyo, 1970 p 29-39; Symposiumon Heat and Polymers, Tokyo, 1971.

4. O. Yamamoto, Polymer J., 2, 509 (1971).

5. H. Kambe, O. Yamamoto, and S. Igarashi, ISAS Report, Institute of Space and Aeronautical Science, University of Tokyo, 1969, No. 438 (Vol. 34, No. 5).
6. K. Ueberreiter and G. J. Kanig, J. Chem. Phys., 18, 399 (1950).

7. W. Knappe, P. Lohe, and R. Wutschig, Angew. Makromol. Chem., 7, 181 (1969).

8. Y. Wada, A. Itami, T. Nishi, and S. Nagai, J. Poly. Sci., Part A-2, 7, 201 (1969).

9. For example, F. Bueche, "Physical Properties of Polymers," Interscience Publishers, New York, N.Y., 1962.

10. L. E. Nielsen, Macromol. Sci.-Revs. Macromol. Chem., C3(1), 69 (1969).

11. A. Chapiro, "Radiation Chemistry of Polymeric Systems," Interscience Publishers, New York, N.Y., 1962.

12. P. Alexander and D. Toms, J. Polym. Sci., 22, 343 (1956).

13. K. Schumacher, Kolloid-Z., 157, 16 (1958).

14. A. Charlesby, "Atomic Radiation and Polymers," Pergamon Press, Oxford, 1960.

15. H. Y. Kang, O. Saito, and M. Dole, J. Amer. Chem. Soc., 89, 1981 (1967).

16. M. B. Fallgatter and M. Dole, J. Phys. Chem., 68, 1988 (1964). 